\title{
Development of the unit for testing the methods of remote computerized monitoring of environmental changes in the "IKI-Monitoring" center for collective use
}

\author{
Evgeny Loupian ${ }^{1,}$, , Viktor Savorskiy ${ }^{1,2}$, Aleksandr Kashnitskii ${ }^{1}$, Dmitrii Kobets ${ }^{1}$, Konstantin \\ Sen'ko ${ }^{1}$, Ivan Balashov ${ }^{1}$ и Ivan Uvarov ${ }^{1}$ \\ ${ }^{1}$ Space Research Institute of the Russian Academy of Sciences, 117997 Moscow, Russia \\ ${ }^{2}$ Kotelnikov Institute of Radioengineering and Electronics of the Russian Academy of Sciences, \\ Fryazino Department 141190 Fryazino, Moscow region, Russia
}

\begin{abstract}
The impact of hazardous waste on people and the environment from agricultural, industrial, military and mining activities form one of the most significant global environmental challenges. These wastes often include heavy metals, acid precipitates (sulfur oxide solutions), hydrocarbons, and other organic chemicals. Creation of the tools of the VEGA-Science service (http://sci-vega.ru/) to control the state of vegetation for the monitoring the impact of technogenic waste and dumps sources (TWDS) on the environment is the main objective of this work. To achieve this goal, proposals for the modernization and development of the VEGA-Science services have been defined to provide the organization and analysis of long-term variability of the vegetation cover caused by the influence of TWDS.
\end{abstract}

\section{Introduction}

The paper [1] gives an analytical review describing the mechanisms of influence of typical harmful TWDS on the vegetation covers and proposes methods and approaches that allow to identify changes in the vegetation cover caused by the influence of TWDS. This work results in the statement of proposals for making up the comprehensive list of parameters for monitoring forest vegetation cover based on satellite observations, including the use of spectral indices for these purposes. The obtained results allowed to substantiate the use of the funds and resources of IKI-Monitoring [2] as a platform for the development of tools to control the impact of TWDS on the environment. The points of the paper [1] underwent the further development resulting in the hereby article.

The main objective of this work is the creation of the tools in framework of the VEGAScience system for assessment of the state of the vegetation cover for monitoring the impact of technogenic waste and dumps sources (TWDS) on the environment. To achieve this goal, proposals for the modernization and development of the VEGA-Science services

\footnotetext{
*Corresponding author: evgeny@d902.iki.rssi.ru
} 
have been defined to provide the organization and analysis of long-term variability of the vegetation cover caused by the influence of TWDS.

The main methodological approach in the work is the object-oriented design method, based on the clarification of user requirements and the development of software services that meet these target requirements.

\section{Requirements for source satellite and auxiliary data for the assessment of the impact of TWDS on the vegetation cover}

The peculiarity of the algorithms for detecting the impact of TWDS is the need to consistently, in a wide range of hydrometeorological parameters, detect the disturbance of vegetation against the background of strong interannual, seasonal and intra-seasonal variability. This necessity is determined by the peculiarities of temperature and humidity conditions, which develop differently in each of the annual cycles, which leads to significant differences in the phenological cycles of plant development. Therefore, the detection of disturbance caused by TWDS, involves the use of such methods of monitoring the state of vegetation, which are resistant to changes in temperature and humidity conditions, in order to ensure long-term monitoring of control areas of forest cover for 1020 years for at least one of the types of disturbance described in the paper [1].

The need to detect the influence of TWDS at significantly different temperature and humidity observation conditions form the following number of requirements for the list of the source and auxiliary data that ensure stable operation of the algorithms for detecting forest canopy disturbance caused by TWDS:

1) TWDS description database (coordinates, type, formation period, polluting factor),

2) database of the forest plots (allotments) having close characteristics and taxonomy (main breeds, stock, bonitet) to the ones in the areas of TWDS,

3) database for search and selection of forest areas (allotments) having similar weather and climatic characteristics (temperature, humidity, soil moisture, wind speed and direction) to the ones in the areas of TWDS,

4) medium and high spatial resolution historical satellite observations data archive for selected control areas of the forest,

5) medium and high spatial resolution real time satellite observations data archive for selected forest areas.

\section{Requirements for the services of detection of the TWDS impact on the vegetation cover based on the satellite observations}

As follows from the analysis, in order to automatically detect the TWDS impact on forest vegetation cover, an automated system for remote monitoring of vegetation cover (SRMV) in the TWDS areas should be launched, being able to provide the following capabilities:

1) service for definition of the list of the forest sites (allocations) having similat taxonomy (basic breeds, stock, bonitet) to the ones in the areas of TWDS,

2) service for definition of the list of forest areas (allotments) having similar weather and climatic characteristics (temperature, humidity, soil moisture, wind speed and direction) to the ones in the areas of TWDS,

3) service for making up the pairs ("observed site" - "reference site") based on the similarity in taxonomy and weather and climatic conditions for each observed TWDS (taking into account the wind rose and proximity of the sites to TWDS), 
4) service for calculation of the spectral indices characterizing the state of the vegetation cover, including NDVI, AVFI1600, DWSI5, EVI, NDNI, OSAVI, PEI, TCARI (see the paper [1] and references) and TIEHD [3, 4], based on medium and high spatial resolution to historical and current satellite observations for pairs ("observed site" "reference site") of forest plots (allotments);

5) service of estimation of difference in spectral indices for the pairs of the sites ("observed site" - "reference site") having the same weather and climatic conditions and having the same taxonomy at the beginning of monitoring,

6) service for analysis of phenological seasonal cycles of pairs of spectral indices for pairs of sites ("observed site" - "reference site") having the same taxonomy at the beginning of monitoring,

7) service for detection and monitoring of long-term cumulative trends of spectral index differences for pairs ("observed site" - "reference site"),

8) service for distinguishability assessment (statistical measure of distinguishability) for pairs ("observed site" - "reference site") having the same weather and climatic conditions, in particular, using the Jeffries-Matsushita distance [5].

All the above algorithms and services should be provided with remote access user web interfaces [6].

\section{The "IKI-Monitoring" Center for Collective Use, the information platform to provide the potential capabilities and resources for solving the problems of monitoring of dumps of technogenic waste}

The requirements listed in sections 2, 3 are substantially satisfied by the information tools developed and implemented in the information system of the IKI-Monitoring CCU (http://ckp.geosmis.ru/). Thus, the VEGA-Science services and tools can be used as prototypes during the development of tools for remote access to the remote forest monitoring data in order to detect forest disturbance due to the impact of TWDS. They provide:

1) accurate geographical reference of the source satellite monitoring data and derived data products, including spectral indices,

2) creation of the TWDS database,

3) automatic and semi-automatic definition of the polygon network,

4) calculation of statistical parameters of the source satellite monitoring data and derived data products, including spectral indices for selected polygons,

5) creation of the database of taxonomically homogeneous forest areas,

6) recognition of homogeneous weather and climatic zones according to long-term hydrometeorological observations,

7) making of spectral index maps, including the ones edited by users in the online mode,

8) creation of the database of seasonal phenological profiles of source satellite monitoring data and derived data products for selected polygons,

9) creation of decadal, monthly and seasonal average values of spectral indices based on long-term satellite monitoring of the polygon network.

The availability of such prototype tools ensures the rapid successful implementation of the TWDS SRMV as a unit of the VEGA-Science system. This is also facilitated by the operation of the IKI-Monitoring center, which includes VEGA-Science systems, information resources of long-term satellite monitoring and related information. The prototype of the specialized unit for remote monitoring of vegetation cover in TWDS areas is currently in operation, providing the implementation of TWDS SRMV as a part of the 
system. An example of the interfaces that provides the operation of this unit is shown in Fig. 1.

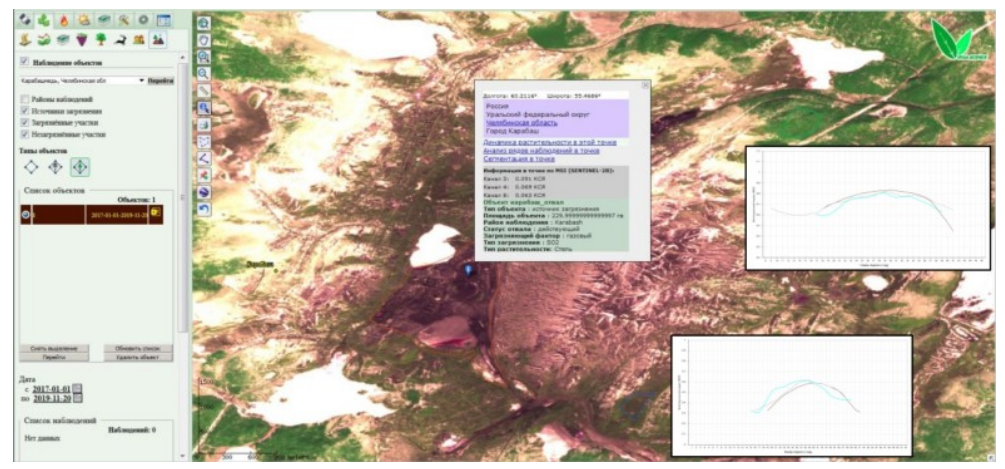

Fig. 1 Interfaces of the specialized unit for remote monitoring of vegetation cover in the TWDS areas. Data analysis in the "Kabashmed" plant dumps area (Chelyabinsk region).

\section{Conclusions}

Proposals for the modernization and improvement of VEGA-Science services, which are necessary for the organization and analysis of long-term variability of vegetation caused by the influence of TWDS, are formulated. In order to test the feasibility of the proposal implementation, a working prototype of a specialized unit for remote monitoring of vegetation cover in the TWDS regions was created as part of the VEGA-Science system.

The study was supported by RFBR in framework of the scientific project No. 18-29$24121 \mathrm{MK}$.

\section{References}

1. V.P. Savorskiy, E.A. Loupian, V.I. Gornyy, D.M. Ermakov, O.Yu. Panova, A.V. Konstantinova, Sovremennye problemy distantsionnogo zondirovaniya Zemli iz kosmosa, 16(6), (2019). (in print)

2. E.A. Loupian, A.A. Proshin, M.A. Bourtsev, A.V. Kashnitskii, I.V. Balashov, S.A. Bartalev, A.M. Konstantinova, D.A. Kobets, A.A. Mazurov, V.V. Marchenkov, A.M. Matveev, M.V. Radchenko, I.G. Sychugov, V.A. Tolpin, I.A. Uvarov, Sovremennye problemy distantsionnogo zondirovaniya Zemli iz kosmosa, 16(3), 151-170 (2019). DOI: 10.21046/2070-7401-2019-16-3-151-170.

3. V.I. Gornyy, S.G. Kritsuk, I.Sh. Latypov, V.N. Khramtsov, Sovremennye problemy distantsionnogo zondirovaniya Zemli iz kosmosa, 10(4), 201-212 (2013).

4. V.I. Gornyy, A.V. Kiselev, S.G. Kritsuk, I.Sh. Latypov, A.A. Tronin, Sovremennye problemy distantsionnogo zondirovaniya Zemli iz kosmosa, 16(4), 124-136 (2019). DOI: 10.21046/2070-7401-2019-16-4-124-136.

5. J.A. Richards, X. Jia, Remote Sensing Digital Image Analysis. Berlin Heidelberg: Springer, (2006), $439 \mathrm{p}$.

6. E.A. Loupian, 1. V.P. Savorskiy, Yu.I. Shokin, A.I. Aleksanin, R.R. Nazirov, I.V. Nedolugko, O.Yu. Panova, Sovremennye problemy distantsionnogo zondirovaniya Zemli iz kosmosa, 9(5), 21-44 (2012). 